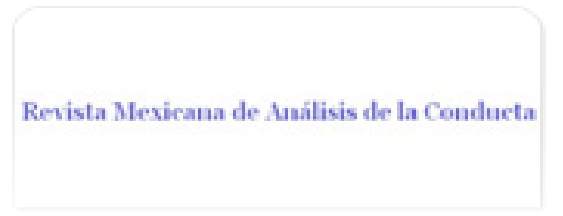

Revista Mexicana de Análisis de la Conducta ISSN: 0185-4534

editora@rmac-mx.org

Sociedad Mexicana de Análisis de la Conducta México

RODRÍGUEZ PÉREZ, MARÍA ELENA; RIBES IÑESTA, EMILIO; VALENCIA VELÁZQUEZ, LILIA MARÍA TRINIDAD; GONZÁLEZ RAMOS, LUIS FERMÍN

Efecto de un entrenamiento observacional con descripciones en la transferencia extradimensional

Revista Mexicana de Análisis de la Conducta, vol. 37, núm. 2, 2011, pp. 155-175

Sociedad Mexicana de Análisis de la Conducta

Guadalajara, México

Disponible en: http://www.redalyc.org/articulo.oa?id=59319255010

Cómo citar el artículo

- Número completo

- Más información del artículo

Página de la revista en redalyc.org

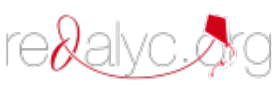

Sistema de Información Científica

Red de Revistas Científicas de América Latina, el Caribe, España y Portugal Proyecto académico sin fines de lucro, desarrollado bajo la iniciativa de acceso abierto 
REVISTA MEXICANA DE ANÁLISIS DE LA CONDUCTA MEXICAN JOURNAL OF BEHAVIOR ANALYSIS

DOI: $10.5514 /$ rmac.v37.i2.26145
2011

$37,155-175$
NUM $2(8-11 / 11-11)$

\title{
EFECTO DE UN ENTRENAMIENTO OBSERVACIONAL CON DESCRIPCIONES EN LA TRANSFERENCIA EXTRADIMENSIONAL
}

\author{
EFFECT OF AN OBSERVATIONAL TRAINING WITH DESCRIPTIONS ON \\ EXTRADIMENSIONAL TRANSFERENCE

\section{MARÍA ELENA RODRÍGUEZ PÉREZ', EMILIO RIBES IÑ̃STA², LILIA MARÍA TRINIDAD VALENCIA VELÁZQUEZ ${ }^{1}$ Y LUIS FERMÍN GONZÁLEZ RAMOS ${ }^{1}$}

\author{
${ }^{1}$ Centro de Estudios e Investigaciones en Comportamiento, Universidad de Guadalajara, \\ Guadalajara, Jalisco, MÉxico \\ ${ }^{2}$ Universidad Veracruzana, Xalapa, Veracruz, México
}

\begin{abstract}
Resumen
Se realizó un estudio experimental con el propósito de evaluar el efecto de un entrenamiento observacional con y sin descripciones en una prueba de transferencia extradimensional. Se utilizaron dos dominios, uno para el entrenamiento y otro para la prueba contrabalanceando el orden de presentación. Los arreglos de igualación de la muestra de segundo orden se armaron utilizando pinturas como instancias considerando uno de los dos dominios: por técnica (cómo se representa lo pintado) o por temática (qué se representa en la pintura). En la prueba, los participantes tuvieron que armar un arreglo de igualación a partir de las mismas instancias de entrenamiento pero considerando ahora el dominio no entrenado. El desempeño en la prueba fue evaluado de manera cualitativa (al reconstruir la ejecución de los participantes en tiempo real) y cuantitativa (al estimar el número de ensayos que se armaron de manera congruente con el nuevo criterio de igualación). Los participantes que describieron tuvieron mejor desempeño en la prueba que quienes no verbali-

La presente investigación fue apoyada por CONACYT a través del pago de becario para investigadores SNI nivel III y el PROMEP a través del fondo de apoyo al establecimiento de Redes Temáticas de Colaboración en su convocatoria de 2008. MERP fue responsable del diseño, análisis de datos y redacción del escrito, ERI supervisó la conducción del estudio, diseño, análisis y elaboración del escrito, LMTVV y LFGR participaron en la aplicación del estudio y en el análisis de datos. Dirigir correspondencia al primer autor: Centro de Estudios e Investigaciones en Comportamiento, Francisco de Quevedo 180, Col. Arcos Vallarta, C.P. 44130, Guadalajara, Jalisco. México, correo electrónico: rpm08428@cucba.udg.mx
\end{abstract}


zaron de manera explícita los criterios de igualación. La construcción de ensayos a partir de un criterio de igualación nuevo parece ser una tarea de ajuste categorial pertinente en el estudio de la conducta sustitutiva referencial.

Palabras clave: Igualación de la muestra de segundo orden, descripciones verbales, transferencia extradimensional, sustitución contingencial.

\begin{abstract}
A research study was carried out to evaluate the effect of an observational training with and without descriptions on an extradimensional transfer test. Two domains were used, one for training and other for testing counterbalancing their order of presentation. Second-order matching-to-sample arrays were built using paintings as instances considering one of two domains: by style (how the painting is represented) or by theme (what it is represented on the painting). During the transfer test session, participants had to build a matching-to-sample array using the same instances but considering the untrained domain. Performance on test was evaluated qualitatively (reconstructing real time performance of participants) and quantitatively (estimating the number of arrays that were congruent with the new matching criterion). Participants who had to describe the matching criteria during training had a better performance on the extradimensional transfer test than those who did not explicitly describe matching criteria. Building matching-to-sample arrays using a new matching criterion seems to be a categorical adjustment task useful when studying referential substitutive behavior.
\end{abstract}

Key words: Second-order matching-to-sample tasks, verbal descriptions, extradimensional transfer test, contingency substitution.

Estudios previos han mostrado la importancia de la participación del lenguaje en la solución de problemas (Goldiamond, 1966; Ribes, 2000; Vygotski, 1934/1997a). En la tradición psicológica cognitiva, se ha supuesto que la acción cognitiva involucra dos niveles de participación del lenguaje: como conocimiento básico en la forma de patrones de acción y como conocimiento estratégico en la forma de metas, objetivos y planes para la acción (Greeno \& Simon, 1988). Una acción (solución al problema) es elegida cuando el individuo tiene una meta la cual es asociada con la acción, la situación estimulativa externa y la información en la memoria de los intentos pasados de alcanzar la meta. Aquí no es el espacio para discutir la pertinencia de esta postura; sólo se resaltara que en dicha propuesta subyace una noción de hombre dualista que nos lleva, erróneamente, a postular que el plan antecede a la acción (Ribes, 2000; Ryle, 1949; Vygostki 1926/1997b). El lenguaje no sólo se refiere a las actividades que toman lugar en el habla, la escritura y la gesticulación sino que constituye un sistema convencional que media y posibilita todas las prácticas humanas (Ribes, 2006). Esta posibilidad reactiva ha permitido fundamentar el surgimiento de las dos funciones 
sustitutivas, la referencial y no referencial, de la taxonomía propuesta por Ribes y López (1985) como formas de organización del comportamiento exclusivamente humano. Es decir, el lenguaje, en tanto sistema reactivo convencional adquirido y con morfología arbitraria respecto de las propiedades "naturales" de los objetos y acontecimientos, permite que el individuo se desligue de las propiedades situacionales (Ribes, 1998). Como el individuo interactúa con dimensiones condicionales a la acción lingüística de otro individuo, se posibilita el desligamiento respecto al aquí, al ahora, lo observable e incluso lo fáctico (Ribes \& López, 1985; Ribes, Ibañez \& Hernández-Pozo, 1986; Vygotski, 1978)

Ribes y López (1985) caracterizaron a la sustitución referencial como un modo de interacción que tiene como elemento crítico mediador a la respuesta de un individuo con respecto a otro o consigo mismo que posibilita reacciones desligadas de las propiedades situacionales aparentes en los eventos, objetos y/u organismos presentes en el ambiente. Por su parte, la sustitución no referencial fue caracterizada por estos autores como una reorganización funcional de contingencias puramente convencionales, es decir, la sustitución de contingencias opera a partir de las propias condiciones de estímulo y respuesta convencionales. Ribes y Zaragoza (2009) han propuesto que la solución de un problema con base en un proceso de sustitución de contingencias puede ocurrir de dos maneras distintas. En una, la sustitución referencial, el individuo tiene que interactuar lingüísticamente respecto de su propio desempeño para responder en momentos ulteriores de la solución del problema, o de un nuevo problema, con base en las propiedades que puede atribuir a la situación dado su desempeño eficaz previo. En la otra, la sustitución no referencial, las distintas fases de un problema determinado, o de nuevos problemas, se traducen de manera sistemática para identificarlos como componentes de una relación más general que los incluye.

Diversos hallazgos experimentales con tareas de igualación de la muestra -como preparación metodológica paradigmática en la investigación de la conducta humana compleja -- constituyen evidencias de que los procesos sustitutivos operan como transformación de las contingencias presentes en una situación a partir de interacciones lingüísticas del individuo que se enfrenta a la solución de un problema. Es decir, la investigación pone de manifiesto el hecho de que las descripciones (en morfologías diversas tales como escribiendo y hablando de lo que se hace; reconociendo y marcando opciones de texto o leyendo y eligiendo opciones de textos que se correspondan a lo que se hizo) sólo son funcionales cuando los sujetos responden a ellas como productos del comportamiento verbal (Ribes, Domínguez, Tena \& Martínez, 1992; Ribes, Torres \& Ramírez, 1996, Ribes \& Serrano, 2006; Ribes \& Zaragoza, 2009). Por ejemplo, se ha encontrado que la posibilidad de responder en las pruebas de transferencia mediante una respuesta descriptiva de la igualación que estaba realizando promovió que los participantes sin entrenamiento previo tuvieran un alto desempeño en transferencia (Cepeda, Hickman, Moreno, Peñalosa, \& Ribes, 1991; Ribes, Domínguez, Tena \& Martínez, 1992; Ribes, Moreno \& Martínez, 1998). 
También se ha encontrado que la anticipación explícita de la respuesta de igualación a ser realizada (mediante un entrenamiento observacional que demandó respuestas verbales explícitas tales como marcar el estímulo de comparación que cree correcto, completar un texto o elegir una justificación antes de recibir la retroalimentación sobre cuál estímulo de comparación era correcto) facilitó el desempeño correcto en entrenamiento y transferencia (Ribes, Rodríguez \& Fuentes, 2003).

Hasta ahora, los criterios empleados para postular la ocurrencia de una interacción sustitutiva en tareas de solución de problemas como la igualación de la muestra han sido el desempeño en pruebas de transferencia y la descripción de los criterios de elección del estímulo comparativo (Ribes, Vargas, Luna \& Martínez, 2009). Por ello, uno de los supuestos con los que se ha trabajado empíricamente es que las diferentes pruebas de transferencia permitirían evaluar cada uno de los niveles de desligamiento del comportamiento (Hurtado, Robayo \& Peña 2007; Ribes \& Serrano, 2006). Sin embargo, no se tiene suficiente evidencia empírica para poder concluir cuáles cambios en las tareas de igualación de la muestra se corresponden con cada uno de los niveles sustitutivos. En un intento por explorar sistemáticamente la posibilidad de estructurar tareas prescribiendo los criterios de ajuste que corresponde a cada una de las funciones propuestas en la taxonomía de Ribes y López (1985), Ribes, Vargas, Luna y Martínez (2009) diseñaron un experimento con exposición secuencial al entrenamiento en interacciones progresivamente complejas. Con esto, se pretendía evaluar el aprendizaje de una función sin el entrenamiento en la función previa y su efecto en la adquisición de la siguiente función. Estos autores encontraron que ninguno de los participantes en los distintos grupos experimentales satisfizo los criterios de adquisición en las funciones sustitutivas. En el caso de la sustitución referencial, los participantes debían comportarse en la tarea como si fuese alguna de las tareas resueltas previamente. Esto implicaba "ver" la tarea lingüísticamente y el exigir respuestas morfológicamente lingüísticas respecto de la respuesta de igualación y sus circunstancias mediante el completado de frases no fue suficiente.

Con la finalidad de evaluar la sustitución referencial de manera directa, en el presente estudio se diseñó una tarea de igualación de la muestra de segundo orden en donde las instancias utilizadas y sus relaciones pudieran ser "vistas" de, al menos, dos maneras diferentes. De esta manera, se podría entrenar en un dominio y probar en el otro si el participante es capaz de comportarse ante el nuevo problema de manera sustitutiva. El entrenamiento fue observacional con la anticipación explícita de la respuesta de igualación correcta y descripciones ya que, en estudios previos, estos procedimientos parecieron promover el desligamiento situacional que supone la sustitución de contingencias. La condición de prueba le demandó al participante construir arreglos de igualación de la muestra a partir de un criterio de ajuste proporcionado por el experimentador. Como control experimental se incluyeron grupos entrenados sin descripciones. Se postularía la ocurrencia de sustitución referencial si el participante lograra un desempeño efectivo durante el entrenamiento en un dominio dado y, además, construyera ensayos de igualación de la muestra que fue- 
ran congruentes con el nuevo criterio de ajuste. Se esperaba que un mayor número de participantes de los grupos que describían mostraran ejecuciones sustitutivas en comparación con los grupos que no describieron.

\section{Método}

\section{Participantes}

Participaron 20 estudiantes universitarios, 14 hombres y 6 mujeres, del área de ciencias exactas e ingenierías cuyas edades variaron entre 20 y 26 años. Ninguno tenía experiencia previa en la tarea experimental. Dado que el conocimiento o la formación en arte pudo haber sido una variable extraña, se les preguntó si contaban con experiencia estética. Ninguno de ellos reportó familiaridad en los dominios evaluados en el presente experimento. Además de este reporte verbal, se estableció como criterio de selección que no alcanzaran más de 24 aciertos durante la preprueba. A cambio de su participación, recibieron créditos en una de las asignaturas que estaban cursando.

\section{Tarea experimental}

Se utilizó una tarea de igualación de la muestra de segundo orden con estímulos pictóricos para igualar según dos dominios distintos: temático o técnica. El dominio temático se definió según lo que está representado en la pintura utilizando cuatro modalidades: bodegón (o naturaleza muerta), paisaje, retrato y escena social (o escena costumbrista). El dominio por técnica se definió según el uso de colores y trazos utilizando cuatro modalidades: cubismo (uso de figuras geométricas en lugar de las formas de los objetos que se representan), puntillismo (uso de puntos de colores puros en lugar de una pincelada uniforme), expresionismo (uso de colores distintos a los colores cotidianos de los objetos representados para comunicar la emotividad del pintor) e impresionismo (representaciones objetivas del mundo). Para armar los arreglos, se localizaron 139 pinturas de varios artistas con el único criterio de que tanto la técnica como la temática fuera fácil de discriminar. En la tabla 1 se muestra un ejemplo de las instancias utilizadas. Tal como puede observarse, cada instancia podía ser identificada en cualquier de los dos dominios. Sin embargo, sólo debía considerarse un dominio al momento de elegir la respuesta correcta.

Los arreglos constaron de seis pinturas acomodados en tres filas (ver figura 1). En la primera fila, los dos estímulos selectores mostraron las relaciones de semejanza o diferencia en un dominio dado. En la segunda fila apareció un estímulo muestra y, en la tercera fila, tres estímulos de comparación ejemplificando las relaciones de identidad, semejanza y diferencia. Las posiciones de los estímulos de comparación y de la respuesta correcta se balancearon para que aparecieran igual número de veces en cada posición de la pantalla. La relación de identidad no se retroalimentó como respuesta correcta en ningún momento del experimento. 
Tabla 1.

Ejemplos de las instancias utilizadas para armar los arreglos de igualación de la muestra de segundo orden.

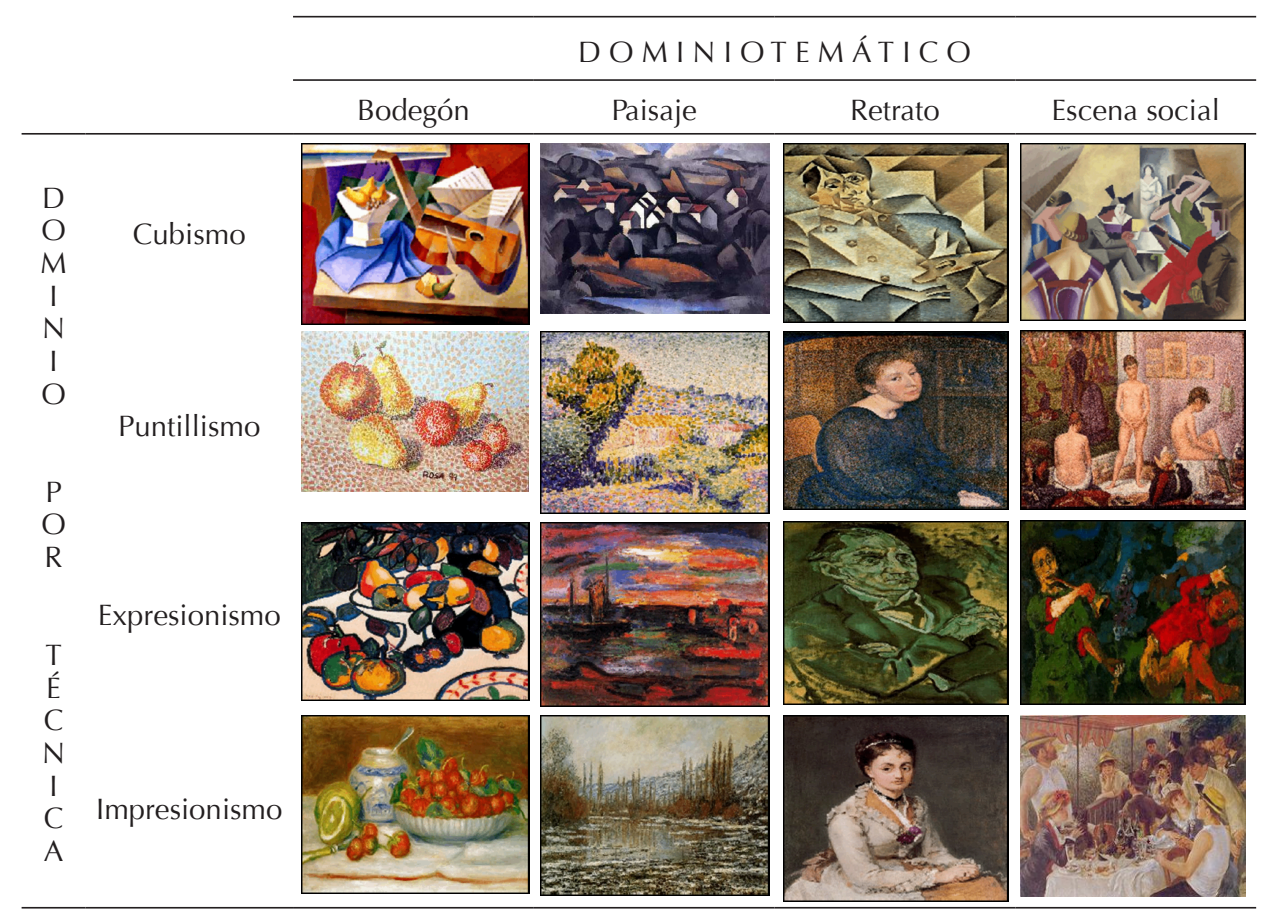

Materiales y aparatos

Las sesiones de preprueba y entrenamiento se realizaron en un salón de $5 \mathrm{~m} \times 6$ $\mathrm{m}$ amueblado con mesas y sillas. Se utilizaron cinco computadoras portátiles en donde se instaló el programa Toolbook Instructor II @ v. 1.5 con el cual se diseñó la tarea experimental y se llevó a cabo el registro de los datos. Los participantes trabajaron de manera individual y sin comunicación entre ellos. Se procuró que no hubiera ruidos ni distracciones que interfiriera con el trabajo de los participantes. Para la sesión de prueba de transferencia se utilizó un equipo HP con pantalla de 23 pulgadas. Esta sesión se realizó en un cubículo amueblado con mesa y silla. La tarea se programó en Visual Basic @ v v. 10 con el que también se grabó la ejecución de los participantes en tiempo real. 


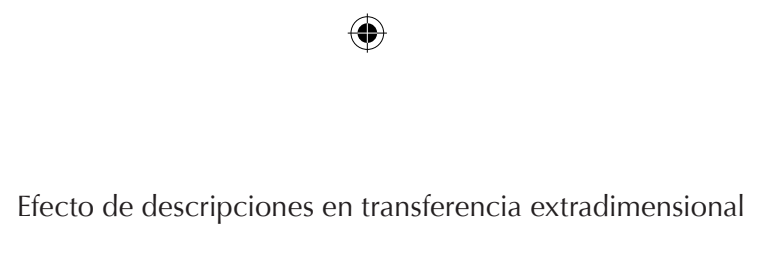

Efecto de descripciones en transferencia extradimensional

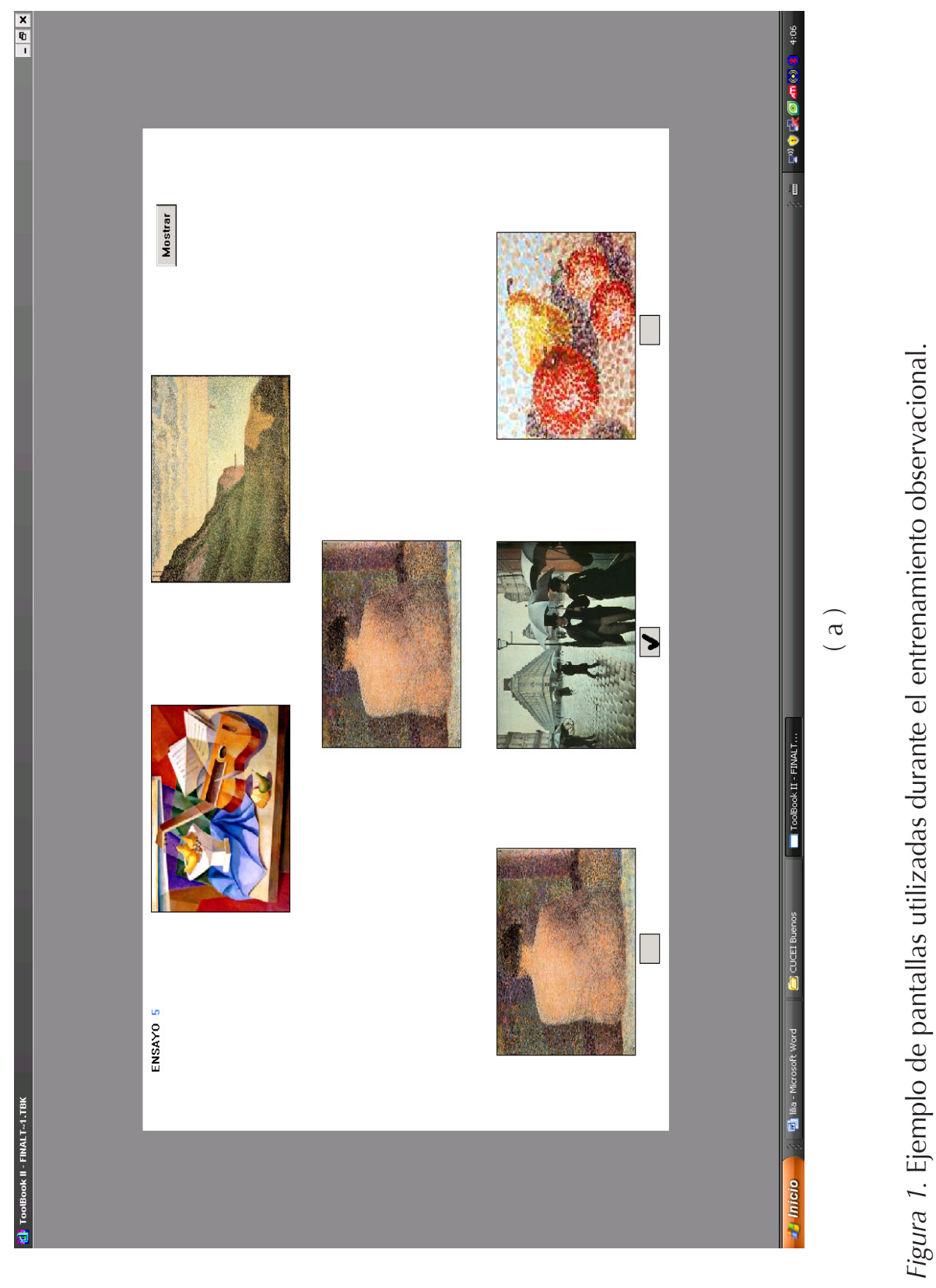

Revista Mexicana de Análisis de la Conducta / Mexican Journal of Behavior Analysis v37 n2 8-2011/11-2011 


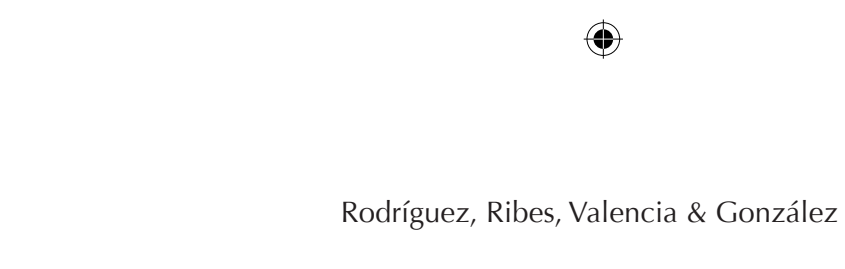

162

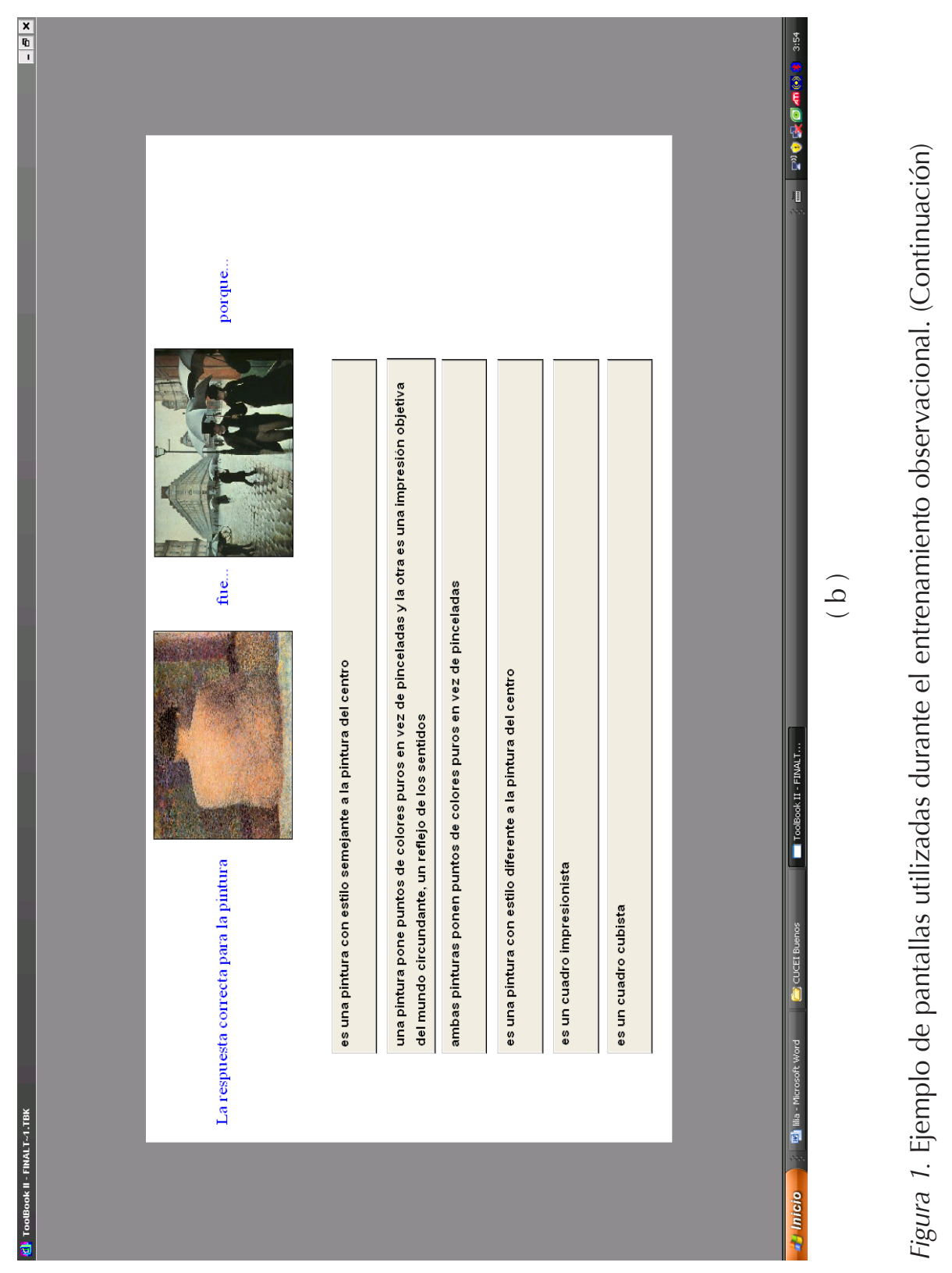

Revista Mexicana de Análisis de la Conducta / Mexican Journal of Behavior Analysis v37 n2 8-2011/11-2011 
Diseño

Se utilizó un diseño experimental con una preprueba, cuatro sesiones de entrenamiento observacional y una sesión para la prueba de transferencia extradimensional (ver tabla 2). Los participantes se asignaron aleatoriamente a uno de los cuatro grupos del experimento por lo que cada grupo constó de cinco participantes. Los grupos controles sólo observaron la retroalimentación respecto de cuál era el estímulo de comparación correcto mientras que los grupos experimentales, después de observar la retroalimentación programada, tuvieron que describir la razón por la cual era la respuesta correcta a través de la elección de opciones de texto. Como control experimental, la exposición a dominios se contrabalanceó para juzgar si éstos fueron equivalentes.

Tabla 2.

Condiciones experimentales a las que fueron expuestos los participantes.

\begin{tabular}{|c|c|c|c|}
\hline Grupo & Preprueba & $\begin{array}{l}\text { Entrenamiento } \\
\text { Observacional }\end{array}$ & $\begin{array}{l}\text { Prueba de transferencia } \\
\text { extradimensional }\end{array}$ \\
\hline Experimental 1 & & $\begin{array}{l}\text { En dominio temático } \\
\text { con descripciones }\end{array}$ & $\begin{array}{l}\text { Construcción de arreglos en } \\
\text { dominio por técnica }\end{array}$ \\
\hline Experimental 2 & Ejecución & $\begin{array}{l}\text { En dominio por técnica } \\
\text { con descripciones }\end{array}$ & $\begin{array}{c}\text { Construcción de arreglos en } \\
\text { dominio temático }\end{array}$ \\
\hline Control 1 & $\begin{array}{l}\text { Instrumental sin } \\
\text { retroalimentación }\end{array}$ & $\begin{array}{l}\text { En dominio temático } \\
\text { sin descripciones }\end{array}$ & $\begin{array}{l}\text { Construcción de arreglos en } \\
\text { dominio por técnica }\end{array}$ \\
\hline Control 2 & & $\begin{array}{l}\text { En dominio por técnica } \\
\text { sin descripciones }\end{array}$ & $\begin{array}{c}\text { Construcción de arreglos en } \\
\text { dominio temático }\end{array}$ \\
\hline Sesiones & 1 & 4 & 1 \\
\hline
\end{tabular}

\section{Procedimiento}

Preprueba

La preprueba se armó con 18 ensayos del entrenamiento en técnica y 18 ensayos del entrenamiento en temática elegidos cuasialeatoriamente para poder contrabalancear la posición de la respuesta correcta y contar con igual número de ensayos de semejanza y diferencia. La ejecución fue instrumental sin retroalimentación.

Entrenamiento observacional sin descripciones

Durante el entrenamiento observacional, los participantes se expusieron a arreglos de igualación de la muestra de segundo orden de temática semejante/diferente o de técnica semejante/diferente según el grupo asignado. Debajo de cada estímulo de comparación aparecía un botón gris (ver figura 1, panel "a"). El participante tenía que marcar cuál creía que era la respuesta correcta. Al hacerlo, aparecía un recuadro con la leyenda "mostrar". Al dar clic sobre este botón, aparecía una flecha o una cruz al lado de cada estímulo de 
comparación según si se tratase de la respuesta correcta o no, con la leyenda "correcta" o "incorrecta" respectivamente. La retroalimentación siempre sucedía en el mismo orden: primero para el estímulo de comparación de la izquierda, después para el del centro y al final para el de la derecha de la pantalla. Al terminar, se pasaba al siguiente ensayo.

\section{Entrenamiento observacional con descripciones}

Este fue similar al entrenamiento observacional pero, antes de pasar al siguiente ensayo, apareció una segunda pantalla para poder describir los criterios de igualación (ver figura 1, panel "b"). En esta nueva pantalla, aparecían las pinturas que se habían utilizado como estímulo muestra y el estímulo de comparación correcto de la pantalla anterior dentro de un enunciado que decía:

"La respuesta correcta para la pintura (pintura de estímulo muestra) fue (pintura del estímulo de comparación correcto) porque..."

Debajo de este enunciado, aparecieron seis opciones de texto. Dos de ellos ponían de relevancia el criterio de semejanza o diferencia por lo que se consideraron descripciones de relación. Otras dos opciones de textos mencionaron (1) la técnica o temática (según el grupo asignado) del estímulo de comparación correcto y (2) una técnica o temática no pertinente para el ensayo en cuestión. Dado que estas descripciones ponían énfasis sólo en la pintura correcta, se consideraron descripciones de instancia. Por último, otras dos opciones de texto mencionaban (1) las modalidades que compartían el estímulo muestra y el estímulo de comparación semejante y (2) las modalidades del estímulo muestra y el estímulo de comparación diferente. Por hacer énfasis en la descripción de los modos de cada dominio, se consideraron descripciones de modalidad. Como puede observarse, en cada ensayo había tres opciones de texto correctos y tres incorrectos según si se tratara de un ensayo de semejanza o de diferencia. El participante debía elegir una opción de texto, la cual era retroalimentada con la palabra "correcto" o "incorrecto" según fuera el caso. Con esto, se pasaba al siguiente ensayo.

Prueba de transferencia extradimensional

Durante la prueba de transferencia extradimensional, los participantes tuvieron que construir arreglos de igualación de la muestra de segundo orden en el dominio no entrenado. Las instrucciones recibidas fueron:

- En esta parte del experimento, aparecerán 8 pinturas en la parte izquierda de la pantalla. Tu tarea consiste en armar arreglos de pinturas que tengan tres filas: en la primera fila deberán aparecer dos pinturas que se relacionan de alguna manera, en la segunda fila deberá aparecer una pintura dada y en la tercera fila deberá aparecer la pintura que tiene la misma relación con la del centro (según las de arriba) y otras dos pinturas que no guarden dicha relación.

- En cada ensayo se te dirá la relación que debes mostrar con las pinturas de arriba. A partir de este enunciado, deberás arrastrar 6 de las 8 pinturas disponibles y colocarlas 
dentro de las áreas punteadas. Enseguida, deberás marcar la pintura de abajo que crees que es la respuesta correcta y describir la razón por la que consideras que es correcta.

- Es importante señalar que los criterios de relación en esta parte del experimento son DISTINTOS a los criterios que se utilizaron anteriormente. En esta parte del experimento, no recibirás ningún tipo de información respecto de tu desempeño.

Así, para cada ensayo se presentó una pantalla dividida en dos secciones (ver figura 2, panel "a"). A la derecha, aparecieron 8 pinturas como estímulos disponibles para trabajar. En la izquierda, había 6 rectángulos grises en tres filas de dos, uno y tres rectángulos respectivamente para poder colocar los estímulos selectores arriba, el de muestra en el centro y los tres estímulos de comparación abajo. El participante debía hacer clic en una de las pinturas y arrastrarla para ponerla en cualquier posición del arreglo de igualación. Debía repetir esta operación hasta llenar los seis rectángulos.

Se tuvo cuidado en elegir las 8 pinturas que se ofrecieron como opciones en cada ensayo de tal manera que se pudiera tener varias formas de construcción de arreglos válidos. Por ello, en un ensayo del dominio temático, había 4 pinturas de un tema en todas las técnicas y 4 pinturas de otro tema en todas las técnicas. En un ensayo del dominio por técnica, 4 pinturas eran de una misma técnica en todas las temáticas y las otras 4 de otra técnica en todas las temáticas. Los pares de técnicas o temáticas se distribuyeron aleatoriamente para que ocurrieran todas las combinaciones posibles en un mismo número de ocasiones y así, no tener sesgo por frecuencia de uso de las instancias.

Debajo de los rectángulos que señalaban las posiciones de los estímulos de comparación, aparecían tres botones de selección. Las instrucciones le decían al participante que debía señalar con estos botones cuál era la respuesta correcta de su arreglo. Al hacerlo, aparecían 8 opciones de texto para describir a la respuesta correcta (dos textos para cada una de las 4 modalidades). El participante podía pasar al siguiente ensayo, al hacer clic en el botón "siguiente ensayo" siempre y cuando estuvieran todas las posiciones del arreglo de igualación llenas, marcada la respuesta correcta y con una opción de texto elegida. La figura $2 b$, presenta un ejemplo del ensayo terminado. Al construir los 36 ensayos, se terminaba la sesión y no recibían información sobre su ejecución.

Como se grabó la ejecución en tiempo real, fue posible analizar cualitativamente la manera en que cada participante le dio sentido al criterio de igualación presentado en cada ensayo. Además, se calificó cada ensayo de la siguiente manera. Se identificó si el participante había colocado dos estímulos selectores que ejemplificaran la relación solicitada. Después, se identificó si esta misma relación existía entre el estímulo muestra y el estímulo de comparación que había marcado como correcto. Enseguida, se identificó si el texto elegido se correspondía en la modalidad pertinente del estímulo de comparación señalado como correcto. Si las tres cosas habían sucedido, se consideró que en ese ensayo el participante había sido congruente con el criterio de igualación proporcionado. Así, para la prueba de transferencia, se contabilizó el número de ensayos con congruencia como una medida cuantitativa de desempeño. 


\begin{abstract}
$\oplus$
Rodríguez, Ribes, Valencia \& González
\end{abstract}

166

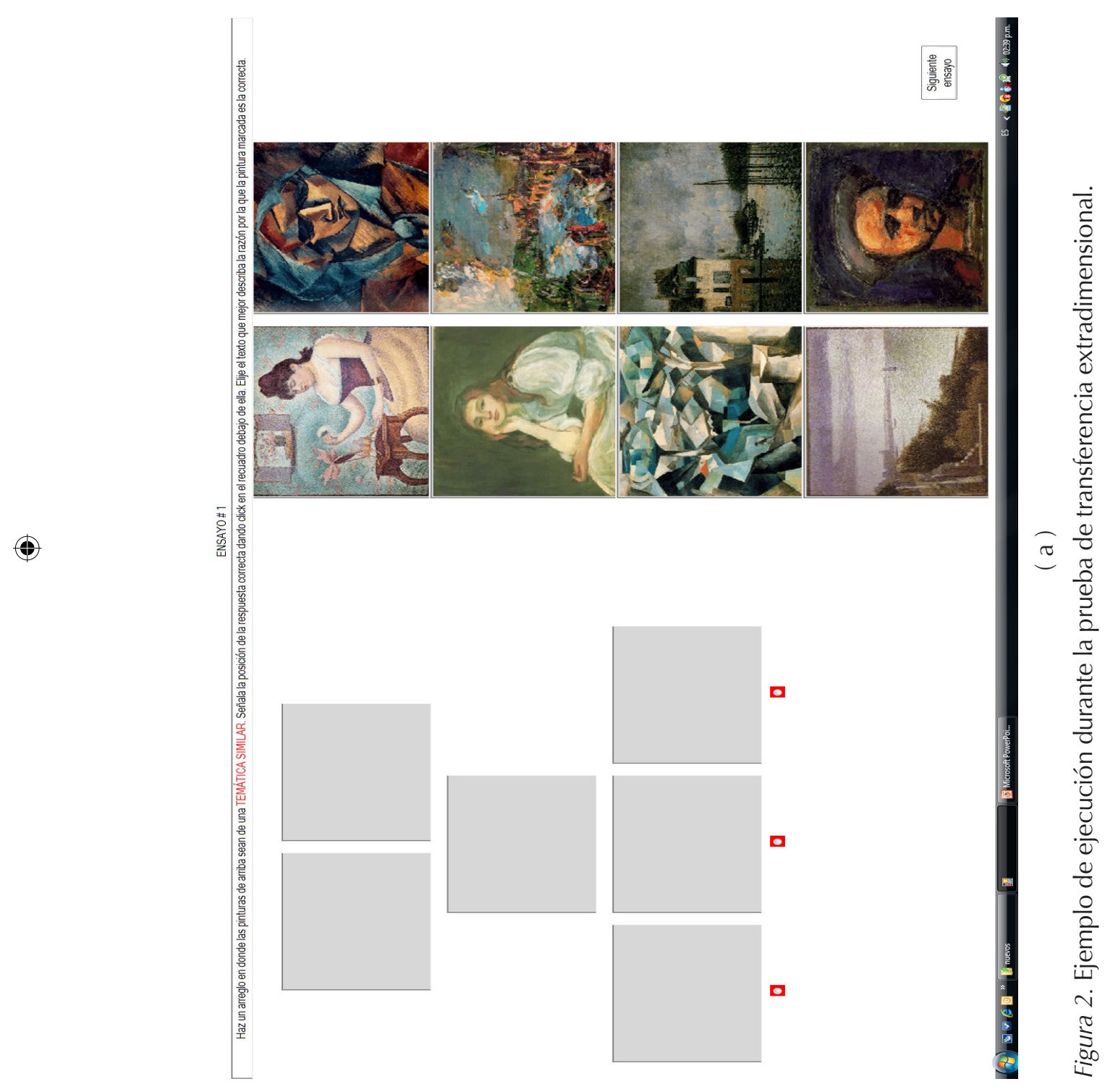

Revista Mexicana de Análisis de la Conducta / Mexican Journal of Behavior Analysis v37 n2 8-2011/11-2011 
Efecto de descripciones en transferencia extradimensional

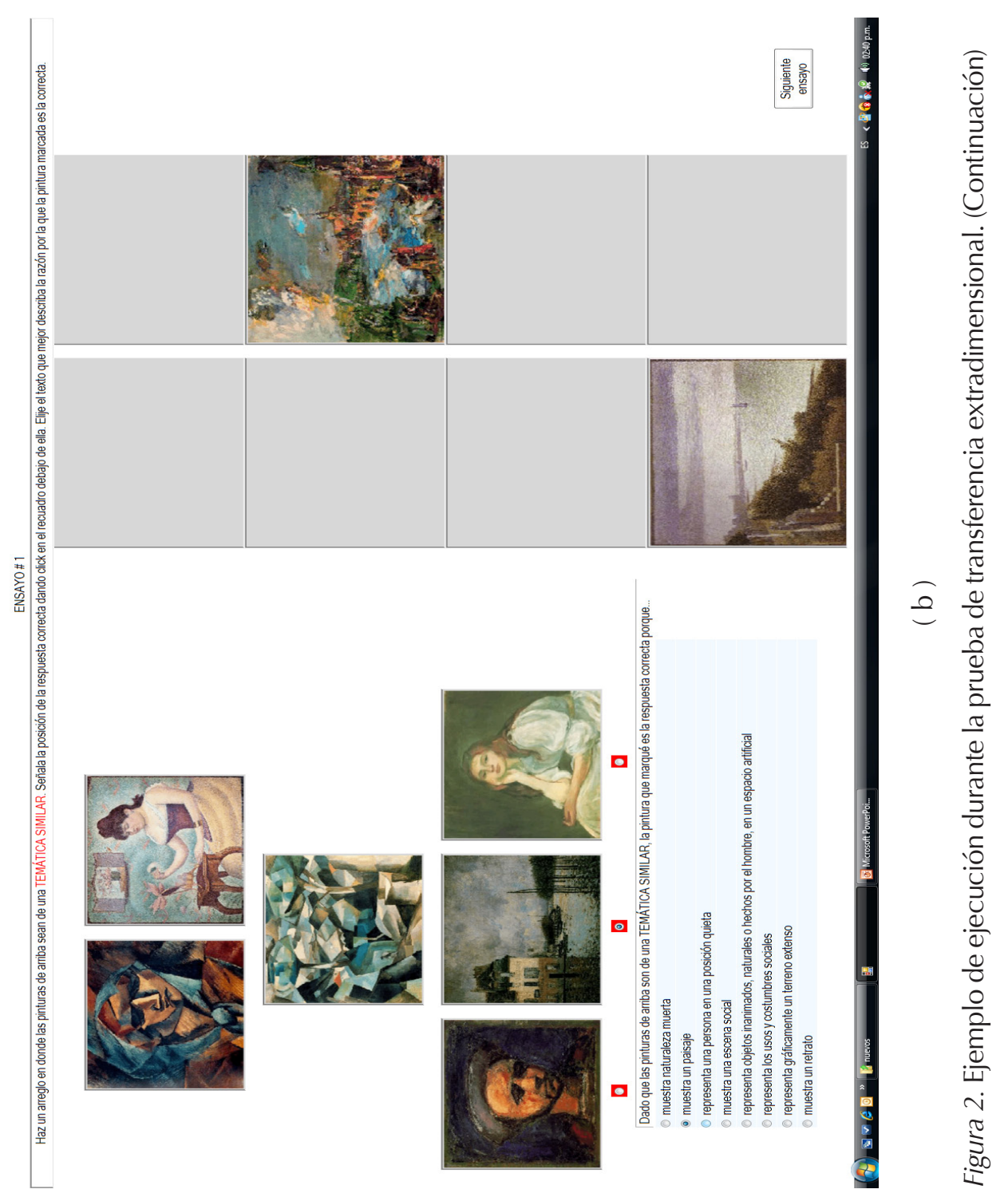

Revista Mexicana de Análisis de la Conducta / Mexican Journal of Behavior Analysis v37 n2 8-2011/11-2011 


\section{Resultados}

La Figura 3 muestra el desempeño de cada uno de los participantes en las diferentes sesiones del experimento. Las barras blancas representan el número de aciertos durante la preprueba mientras que las barras negras muestran el número de ensayos que los participantes construyeron de manera congruente durante la prueba de transferencia extradimensional. Para las cuatro sesiones de entrenamiento, se reporta el número de ensayos en donde el participante marcó la respuesta correcta (cuadrados unidos con una línea) antes de recibir retroalimentación al respecto. Para los grupos experimentales, también se muestra el número de descripciones correctas que tuvieron en cada sesión de entrenamiento (barras con patrón). El tipo de descripción está señalado con patrones diferentes.

De la figura 3, se observa que el número de aciertos durante la preprueba no fue mayor de 24, criterio establecido para seleccionar los participantes y, con ello, suponer que los efectos de las variables manipuladas no pueden atribuirse a una formación estética previa. Los grupos experimentales (que describieron) presentaron un mejor desempeño que los grupos controles (sin descripciones) con respecto al número de ensayos que marcaron correctamente antes de recibir retroalimentación durante el entrenamiento observacional. Ocho de los diez participantes que describieron terminaron con más de 30 aciertos (considerando que un buen criterio de desempeño puede ser arriba del $80 \%$ de respuestas correctas) mientras que sólo la mitad de quienes no describieron lograron un desempeño similar.

Todos los participantes de los grupos experimentales terminaron con más de 34 ensayos con descripciones correctas. El tipo de descripción utilizada fue variable entre los participantes de ambos grupos. Los participantes P1, P3, P4, P5 y P10 utilizaron opciones de textos que hacían referencia a las modalidades de las pinturas. Los participantes P6, P7, P8 y P9 utilizaron opciones de texto que ponían de relevancia el criterio de semejanza o diferencia. El participante P2 utilizó descripciones del tipo de relación para los ensayos de igualación por diferencia y descripciones del tipo de modalidad para los ensayos de igualación por semejanza. La mayoría de los participantes no cambiaron el tipo de descripción utilizada a lo largo de las cuatro sesiones del entrenamiento. El desempeño en la prueba de transferencia pareció ser independiente del tipo de descripción o de la ejecución durante el entrenamiento. A partir de la figura 3, podemos observar que los grupos experimentales tuvieron un mejor desempeño durante la prueba de transferencia extradimensional que los grupos controles. Uno de los cinco participantes del grupo 1 (entrenamiento en el dominio temático y prueba en el dominio por técnica) y cuatro de los cinco participantes del grupo 2 (entrenamiento en el dominio por técnica y prueba en el dominio temático) lograron tener más de 24 ensayos (67\%) con congruencia durante la condición de prueba. Esto no ocurrió con ninguno de los participantes de los grupos controles. El mejor desempeño de los participantes del grupo 2 puede deberse a que es más fácil para un lego discriminar contenidos que procedimientos. 
Efecto de descripciones en transferencia extradimensional

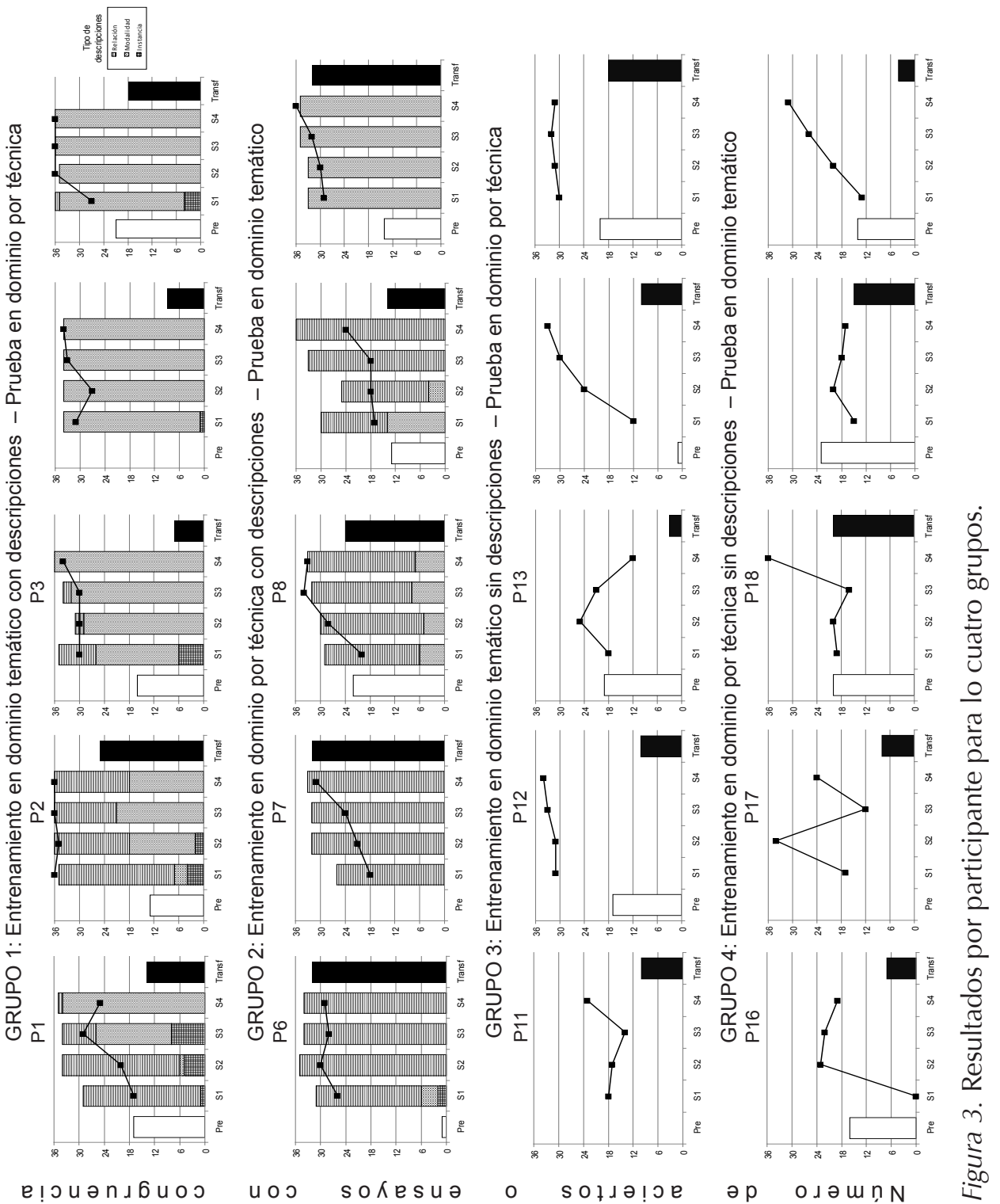

Revista Mexicana de Análisis de la Conducta / Mexican Journal of Behavior Analysis v37 n2 8-2011/11-2011 
La tabla 3 resume el desempeño durante la prueba de transferencia en cada aspecto que se consideró para calificar la congruencia. Se ha resaltado el número más bajo ya que éste sería el factor limitante para poder construir los arreglos de manera congruente con el criterio de igualación proporcionado. En algunos casos, fue posible describir ejecuciones sistemáticas como desempeños idiosincrásicos, lo cual es evidencia de que los participantes no estuvieron resolviendo la tarea de manera azarosa. En un análisis minucioso de la figura 3 y la tabla 3, podemos ver que el participante del grupo 1 con el mayor número de ensayos con congruencia (P2) es aquel que utilizó de manera diferencial las opciones de texto durante el entrenamiento observacional según si se tratara de un ensayo de igualación por semejanza o diferencia. En este mismo grupo, fue posible identificar una ejecución sistemática pero incorrecta para el participante P4 (con sólo 9 ensayos con congruencia). Este participante eligió pinturas que ejemplificaran una relación de semejanza en temática (dominio entrenado) para las posiciones de los estímulos de segundo orden y armó correctamente un arreglo de igualación de la muestra de primer orden con el criterio de diferencia en técnica. Parece que el uso de los textos fue al azar y que, precisamente, esta dificultad para nombrar a los estímulos de comparación que marcó como correctos fue el factor limitante para cumplir correctamente con el criterio de igualación proporcionado. Para los participantes P1 y P3 del grupo 1, el factor limitante fue la construcción del arreglo de primer orden mientras que el participante P5 utilizó de manera equivocada las opciones de texto "es un cuadro expresionista" y "es un cuadro impresionista". Con respecto al grupo 2, el único participante que tuvo un bajo número de ensayos con congruencia (P9) armó correctamente el arreglo de primer orden en el nuevo dominio y usó de manera adecuada las opciones de texto para describir la respuesta marcada como correcta. El factor limitante fue la elección de los estímulos selectores. Este participante mostró una ejecución idiosincrásica ya que en los ensayos en donde se le pidió que armara un arreglo de "temática diferente" eligió pinturas que fueron diferentes tanto en temática como en técnica mientras que en los ensayos de "temática similar" utilizó pinturas que diferían sólo en la temática o en la técnica. Con respecto a los grupos controles, se encontraron ejecuciones sistemáticas aunque incorrectas como la del participante P19 quien siempre eligió estímulos selectores que mostraran el criterio de semejanza. Llama la atención el desempeño de los participantes P11 y P15 quienes señalaron de manera adecuada la relación del nuevo criterio con los estímulos selectores y describieron bien pero no construyeron el arreglo de primer orden de manera consistente.

\section{Discusión}

En el presente estudio, los participantes se entrenaron en una tarea de igualación de la muestra de segundo orden con estímulos pictóricos en un dominio y se probaron en un dominio distinto con el propósito de postular interacciones sustitutivas referenciales en aquellos casos donde la construcción de arreglos de igualación a partir de las mismas instancias entrenadas mostraran ser congruentes con los nuevos criterios 
Efecto de descripciones en transferencia extradimensional

Tabla 3.

Calificaciones para los tres aspectos que definieron un ensayo con congruencia durante la prueba extradimensional. El total de ensayos fue de 36. Se ha sombreado la calificación más baja para señalar el aspecto que limitó la ejecución de algunos participantes.

\begin{tabular}{|c|c|c|c|c|c|}
\hline \multirow[t]{2}{*}{ Grupo } & \multirow[t]{2}{*}{ Participante } & \multicolumn{3}{|c|}{$\begin{array}{c}\text { Número de ensayos que cumplen con el criterio } \\
\text { de igualación según }\end{array}$} & \multirow{2}{*}{$\begin{array}{l}\text { Número de } \\
\text { ensayos con } \\
\text { congruencia }\end{array}$} \\
\hline & & ASPECTO 1 & ASPECTO 2 & ASPECTO 3 & \\
\hline \multirow{5}{*}{1} & P1 & 23 & 19 & 26 & 14 \\
\hline & $\mathrm{P} 2$ & 33 & 29 & 32 & 25 \\
\hline & P3 & 23 & 12 & 23 & 7 \\
\hline & P4 & 19 & 19 & 12 & 9 \\
\hline & P5 & 32 & 31 & 21 & 18 \\
\hline \multirow{5}{*}{2} & P6 & 33 & 33 & 35 & 32 \\
\hline & P7 & 35 & 33 & 33 & 32 \\
\hline & P8 & 30 & 28 & 33 & 24 \\
\hline & P9 & 19 & 28 & 31 & 14 \\
\hline & $\mathrm{P} 10$ & 33 & 33 & 36 & 32 \\
\hline \multirow{5}{*}{3} & P11 & 33 & 16 & 25 & 10 \\
\hline & $\mathrm{P} 12$ & 18 & 17 & 27 & 10 \\
\hline & $\mathrm{P} 13$ & 19 & 19 & 6 & 3 \\
\hline & P14 & 21 & 21 & 18 & 10 \\
\hline & P15 & 36 & 20 & 31 & 18 \\
\hline \multirow{5}{*}{4} & P16 & 19 & 16 & 22 & 7 \\
\hline & $\mathrm{P} 17$ & 17 & 17 & 27 & 8 \\
\hline & P18 & 26 & 25 & 27 & 20 \\
\hline & P19 & 19 & 25 & 34 & 15 \\
\hline & P20 & 17 & 14 & 27 & 4 \\
\hline
\end{tabular}

Aspecto 1. Los estímulos selectores ejemplifican la relación demandada.

Aspecto 2. El estímulo muestra y el estímulo de comparación marcado como correcto ejemplifican la relación demandada.

Aspecto 3. La opción de texto se corresponde con la temática o la técnica del estímulo de comparación marcado como correcto.

Revista Mexicana de Análisis de la Conducta / Mexican Journal of Behavior Analysis v37 n2 8-2011/11-2011 
de igualación. Los resultados sugieren que la mitad de los participantes entrenados con descripciones lograron un comportamiento sustitutivo. Esto no ocurrió con los participantes de los grupos que no describieron durante el entrenamiento. El tipo de descripciones que utilizaron quienes parecen haberse comportado de manera sustitutiva fue variable. Por tanto, parece que el elemento clave para lograr un alto número de ensayos con congruencia fue el hecho de describir sin importar el contenido de dichas descripciones. Este hallazgo es congruente con estudios previos en donde el contenido formal de una descripción no es el responsable del desempeño en la discriminación condicional (Ribes, Moreno \& Martínez, 1995; Ribes, Torres \& Ramírez, 1996).

En la literatura de corte cognitiva se ha reportado que el entrenamiento observacional en tareas de solución de problemas puede ser tan efectivo como el entrenamiento instrumental (Osman, 2008). También se ha encontrado que, en tareas que demandan habilidades perceptuales o aprendizaje verbal que no dependa de la memorización, la práctica de dicha habilidad en contextos variados promueve una mayor oportunidad para "procesar" (sic.) los rasgos distintivos de la solución al problema y, con esto, promover una mejor transferencia de la solución a otras tareas (Goode, Geraci \& Roediger, 2008). En el presente estudio, el entrenamiento observacional fue más efectivo cuando se acompañó de la elección de textos descriptivos de las razones por las cuales el estímulo señalado era correcto. Esto sugiere que el entrenamiento observacional puede ser tan efectivo como el instrumental porque se "ve" la tarea "lingüísticamente"; ofreciendo un soporte empírico a la noción de que el lenguaje está entremezclado con la acción (Ribes \& López, 1985; Vygotski, 1978) y no en forma de planes que anteceden a la acción. Vygostki (1978) ha argumentado que la planeación de la acción ocurre como la forma más evolucionada del lenguaje interiorizado y como resultado de la independencia de los elementos situacionales. El que ocurra una mayor transferencia cuando se practica en contextos variados presupone que se tenga que describir ejecuciones diferentes como casos de una misma solución; es decir, favorece una ejecución "racional" (Bennet, ), una conducta "orientada a metas" (Vygotski, 1978) o desempeños funcionales "sustitutivos" (Ribes \& López, 1985).

Un hallazgo interesante del presente estudio es el alto número de participantes que lograron ajustarse a los criterios de la prueba de transferencia extradimensional, en particular, los que se entrenaron en el dominio por técnica y se probaron en el dominio temático. Este hecho llama la atención dado que en investigaciones previas se ha reportado que nadie o casi nadie puede ajustarse a los criterios de tareas que demandan un desligamiento extrasituacional (Cortés, Romero \& Hernández, 2002; Mares, Guevara, Rueda, Rivas \& Rocha, 2004; Pérez-Almonacid \& Suro, 2009; Ribes, Vargas, Luna \& Martínez, 2009). Una posible explicación para estas diferencias radica en los procedimientos empleados. Puede ser que se está denominando como "sustitución referencial" a interacciones de naturaleza distinta. No hay que olvidar que las funciones sustitutivas de contingencias requieren de interacciones lingüísti- 
cas para tener lugar pero no deben confundirse con interacciones que ocurren con morfologías lingüísticas (Ribes, Vargas, Luna \& Martínez, 2009). Por tanto, es necesario encontrar preparaciones experimentales que permitan postular la sustitución contingencial de manera fehaciente y que se usen regular y sistemáticamente en el estudio del comportamiento sustitutivo.

La exigencia de construir los ensayos de igualación, el uso de instancias que pueden ser igualadas de acuerdo a diferentes dominios y la combinación del análisis cualitativo y cuantitativo de los datos generados reveló aspectos conductuales ligados al hecho de ver la tarea lingüísticamente. Esto no se reduce a la descripción de las instancias sino que implica el reconocimiento de los criterios de pertinencia con que se acomodan las instancias en los espacios permitidos, las relaciones establecidas entre las instancias, los criterios de relación en operación en un momento dado de la tarea, etcetera. Es decir, se trata de un "juego de lenguaje" (Wittgenstein, 1953). En una revisión crítica del procedimiento empleado para la condición de prueba en la presente investigación, se podría considerar que es una tarea de ajuste categorial. Ribes (2007) establece que el ajuste categorial es todo ajuste posibilitado por un medio de contacto convencional el cual está constituido por redes de prácticas institucionales, objetos y objetos convencionales (naturales y simbólicos) que posibilitan la convivencia como una determinada forma de vida social y sus criterios (o "juego de lenguaje"). Por tanto, las categorías y conceptos, como entidades lingüísticas, delimitan la pertinencia funcional del comportamiento en situación. El individuo humano se ajusta siempre a criterios categoriales (lo que es, lo que pertenece, lo que es apropiado). La tarea, modificada de esta manera, puede ser útil en el estudio de la conducta sustitutiva. En futuros estudios, se incluirá la construcción de arreglos de igualación en la fase de entrenamiento para contrastarlo con el desempeño en pruebas.

\section{Referencias}

Bennett, J. (1964) Rationality: An essay toward analysis. London: Routledge \& Kegan Paul Limited.

Cepeda, M. L., Hickman, H., Moreno, D., Peñalosa, E., \& Ribes, E. (1991) The effect of prior selection of verbal descriptions of stimulus relations upon the performance in conditional discrimination in human adults. Revista Mexicana de Análisis de la Conducta, 17, 53-79.

Cortés, A., Romero, P., \& Hernández, R. (2002) Interacciones niño-niño en tareas de simulación: La importancia de la historia de mediación. Universitas Psychologica, 1(1), 7-18.

Goldiamond, I. (1966) Perception, language and conceptualization rules. En B. Kleinmuntz (Ed.), Problem solving: Research, method and theory (pp. 183-224). New York: Wiley.

Goode, M. K., Geraci, L., \& Roediger, H. L. (2008) Superiority of variable to repeated 
practice in transfer on anagram solution. Psychonomic Bulletin \& Review, 15, 662-666. Disponible vía: http://dx.doi.org/10.3758/PBR.15.3.662

Greeno, J. G., \& Simon, H. A. (1988) Problem solving and reasoning. En R. C. Atkinson, R. J. Herrnstein, G., Lindzey, \& R. D. Luce (Eds.). Steven's Handbook of Experimental Psychology [2a Ed.] (pp. 589-671). New York: Wiley \& Sons.

Hurtado, C., Robayo, M. A., \& Peña, T. E. (2007) Efectos en la ejecución durante una tarea de igualación a la muestra según el tipo y el orden de exposición a las pruebas de transferencia. Universitas Psychologica, 6 (2), 425-440.

Mares, G., Guevara, Y., Rueda, E., Rivas, O. \& Rocha, H. (2004). Análisis de las interacciones maestra-alumnos durante la enseñanza de las ciencias naturales en primaria. Revista Mexicana de Investigación Educativa, 9(22), 721-745.

Osman, M. (2008) Seeing is as Good as Doing. Journal of Problem Solving, 2(1), 29-40.

Pérez-Almonacid, R., \& Suro, A. (2009) Relación entre la historia extrasituacional y el desempeño en pruebas de ajuste transituacional. Revista IPyE: Psicología y Educación, 3(6), 20-46.

Ribes, E. (1998) El lenguaje desde la perspectiva del estudio del comportamiento: Un análisis interconductual. En V. M. Alcaraz (Coord.), Una mirada múltiple sobre el lenguaje: Una perspectiva multidisciplinaria (pp. 225-239). México: Universidad de Guadalajara.

Ribes, E. (2000) Instructions, rules and abstraction: A misconstrued relation. Behavior and Philosophy, 28, 41-55.

Ribes, E. (2006) Conceptos, categorías y conducta: reflexiones teóricas. Revista Latina de Pensamiento y Lenguaje, 15(1), 5-23.

Ribes, E. (2007) Estados y límites del campo, medios de contacto y análisis molar del comportamiento: Reflexiones teóricas. Acta Comportamentalia, 15(2), 229-259.

Ribes, E., Domínguez, M., Tena, O., \& Martínez, H. (1992) Efecto diferencial de la elección de textos descriptivos de contigencias entre estímulos antes y después de la respuesta de igualación en una tarea de discriminación condicional. Revista Mexicana de Análisis de la Conducta, 18, 31-59.

Ribes, E., Ibáñez, C., \& Hernández-Pozo, R. (1986) Hacia una psicología comparativa: Algunas consideraciones conceptuales y metodológicas. Revista Latinoamericana de Psicología, 18, 263-276.

Ribes, E., \& López, F. (1985) Teoría de la conducta. Un análisis de campo y paramétrico. México: Trillas.

Ribes, E., Moreno, D., \& Martínez, C. (1995) Interacción del entrenamiento observacional e instrumental con pruebas de transferencia verbales y no verbales en la adquisición y mantenimiento de una discriminación condicional. Revista Mexicana de Análisis de la Conducta, 21, 23-45.

Ribes, E., Moreno, D., \& Martínez, C. (1998) Second-order discrimination in humans: The roles of explicit instructions and constructed verbal responding. Behavioural Processes, 42, 1-18. Disponible vía: http://dx.doi.org/10.1016/ S0376-6357(97)00056-9 
Ribes, E., Rodríguez, M. E., \& Fuentes, T. (2003) Anticipating the correct matching response in a second-order matching-to-sample task. Psychological Reports, 93, 1307-1318. Disponible vía: http://dx.doi.org/10.2466/PR0.93.8.1307-1318

Ribes, E., \& Serrano, M. (2006) Efectos de tres tipos de pre-entrenamiento en la adquisición y transferencia de una tarea de igualación de la muestra. Acta Comportamentalia, 14, 145-169.

Ribes, E., Torres, C., \& Ramírez, L. (1996) Efecto de los modos de descripción en la adquisición y transferencia de una discriminación condicional de segundo orden en humanos adultos. Acta Comportamentalia, 4, 159-178.

Ribes, E., Vargas, I., Luna, D., \& Martínez, C. (2009) Adquisición y transferencia de una discriminación condicional en una secuencia de cinco criterios distintos de ajuste funcional. Acta Comportamentalia, 17(3), 299-331.

Ribes, E., \& Zaragoza, A. (2009) Efectos de las instrucciones y descripciones con sin criterios en la adquisición y transferencia de una discriminación condicional de segundo orden. Acta Comportamentalia, 17(1), 61-95.

Ryle, G. (1949) The concept of mind. Chicago, Illinois: The University of Chicago Press. Vygotski, L. S. (1978) El desarrollo de los procesos psicológicos superiores. (Trad. al cast. 1979) España: Crítica. (Trabajo publicado a partir de escritos inéditos)

Vygotski, L. S. (1997a) Los métodos de investigación reflexológicos y psicológicos. En A. V. Zoporózhets (Ed.), Liev Semiónovich Vygotski: Obras Escogidas (pp. 3-22). Tomo I. (Trad. al cast. 1991) España: Visor. (Trabajo original publicado en 1926)

Vygotski, L. S. (1997b) Pensamiento y Lenguaje. En A. V. Zoporózhets (Ed.), Liev Semiónovich Vygotski: Obras Escogidas (pp. 9-348). Tomo II. (Trad. al cast. 1991) España: Visor. (Trabajo original publicado en 1934)

Wittgenstein, L. (1953) Philosophical Investigations. Oxford: Basil Blackwell. 